\title{
Kit of Self-Moving Units for Automatic Inspection of Marine Structures and Plants: Low Cost Unit for Inspection of Holds
}

\author{
Enrico Ravina \\ Department of Electrical, Electronic, Telecommunication Engineering and Naval Architecture, Polytechnic School, University of \\ Genoa, Via Montallegro 1, Genoa, Italy
}

\begin{abstract}
All vessels and offshore units require regular inspections to prevent structural or functional damages. Periodical inspections are based on classification society's requirements and include both visual surveys and non-destructive test interventions, to look for structural damage, pitting, corrosion, thickness measurement, usually documented by measurements or images. Different approaches of inspection can be proposed for underwater and for dry areas, using unmanned vehicles or service robots able to improve traditional inspection methods. Several excellent proposals are today available: nevertheless, reasons of costs, operation complexity and impossibility to cover all types of inspection with a unique autonomous unit limit and conditions the applicability of automatic inspection systems. The paper is the first of a series describing the main results of a wide research activity carried out at DREAMS Lab (Laboratory of Drives and Experimental Automation for Marine Systems) of the University of Genoa. The studies are oriented to the realization of a kit of low cost and unsophisticated self-moving units, devoted to support some recursive inspections in dry areas of ships and on offshore plants as bulkheads, holds, double bottoms and ducts. The paper describes the design and prototyping of a pneumatronic unit oriented to inspection and maintenance of ship holds. Conceived to realize a portable and user-friendly tool which could take part of an "inspection kit" for the use of inspectors, able to simplify and speed up the inspection visits with and automatic generation of survey reports.
\end{abstract}

Key words: Self-moving, marine inspection, non-destructive testing.

\section{Introduction}

Traditional marine inspections in dry areas require preliminary expensive and time-consuming interventions before the proper inspection process. Installations of scaffolding (Fig. 1) allowing the surveyor to inspect structures as bulkheads, beams and stiffeners are included in traditional approaches. As an alternative, aerial articulated platforms of various geometry can be employed, transporting the surveyor by a tower crane (Fig. 2). In any case, the proper inspection is manual, with access of the inspector in uncomfortable, critical, or potentially dangerous areas.

In order to reduce costs and time consumption and to

Corresponding author: Ravina Enrico, professor, research fields: drives, fluid power automation and mechatronics for marine systems. improve and speed up the technical surveys, several proposals of autonomous and robotized systems for marine vessel inspection are proposed. Some of these have been inspired by climbing robots designed for general locomotion and adhesion tasks; others are customized for ship areas. A great number of different types of devices for locomotion on surfaces have been designed, and are applicable to general-purpose inspections inside ships:

- legged, implementing motion by the adoption of legs $[1,2]$;

- wheel-driven, combining wheels for translation/ rotation and vacuum pumps/magnets for surface attachment [3, 4];

- Tracked, using chain-tracks as the locomotive mechanism [5]; 


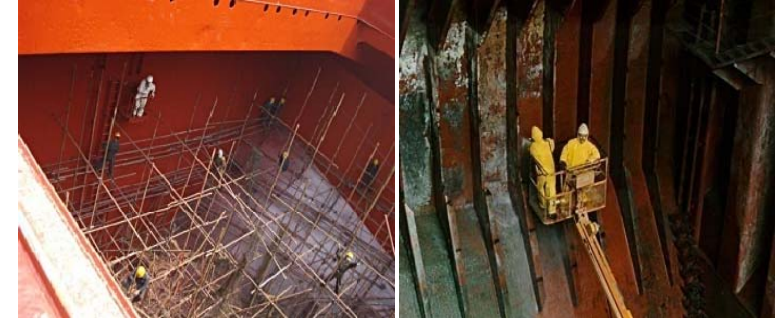

Fig. 1 Scaffolding and articulated platform.

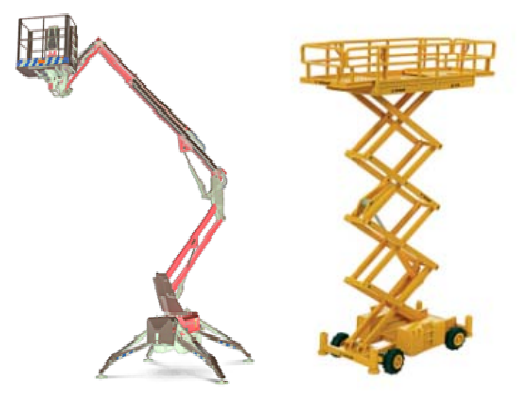

Fig. 2 Models of articulated platforms.

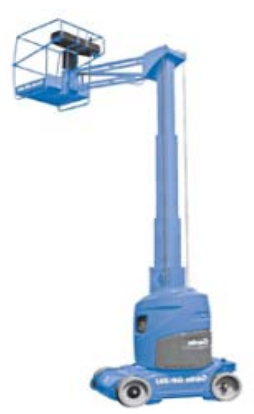

- translation, using translational actuators with appropriate attachment devices $[6,7]$;

- cable-driven [8];

- combined, integrating different locomotive mechanisms to improve the robot ability and dexterity $[9,10]$.

From the surface adhesion point of view, the types proposed consider:

- suction, using vacuum suction cups $[11,12]$;

- magnetic, employing permanent or electrical magnetization devices [13, 14];

- gripping, in which gripping devices allow the climbing robot to travel within complex environments $[15,16]$;

- rail-guided, pre-installing rails for the sliding of the robot $[17,18]$;

- bio-mimetic, emulating, for instance, the sticking ability of geckos $[19,20]$.

It must be observed that not all the adhesion proposals can be successfully applied in dry surface inspection of ship holds.

Inspection and maintenance is a very real problem for classification societies, marine service providers, ship-owners, assurance societies and it represents one of the main elements for the safety and functionality of ships and offshore units. In particular, cargo holds and tanks are subject to regular inspections and maintenance measurements: many spaces are difficult to access and tanks are often narrow. Cargo holds are larger spaces, but they reach easily heights over ten meters; the use of scaffolding forces up not only the costs but also, considering the time required to built the infrastructure, the overall inspection time. From this point of view an automatic inspection process can speed up the intervention. On the contrary, ship inspections are often still done manually and the reason is that the ship environments are, in general, difficult for mobile inspection units.

There are not general criteria of judging or deciding whether automatic units are certain to have advantages on inspection speed over traditional approaches. However, depending of the case, a careful analysis on the different expense items allows evaluating the effective convenience to employ automatic approaches. Considering the specific field of application, it is not realistic to propose the use of a single mobile unit for inspections of all the ship and off-shore spaces: but kits of portable units can be designed to satisfy different requirements.

EU-funded research projects (MINOAS, Marine INspection rObotic Assistant System, or INCASS, inspection CApabilities for enhanced Ship Safety) address this challenge in an attempt to develop concepts for the automation of the ship inspection process. These multifaceted projects integrate experienced and dedicated partners in order to tackle the issue of ship inspection, aiming to avoid ship accidents, promote maritime safety, and protect the environment. Within these projects original climbing units, as Clawer and Clawer II [21, 22], based on magnetic adhesion, have been designed and realized.

At the DREAMS Lab (Laboratory of Drives and Experimental Automation for Marine Systems) of the University of Genoa (Italy), are under continuous development research activities oriented to low cost and compact multi-functional climbing robots, 


\section{Low Cost Unit for Inspection of Holds}

supporting inspection survey and certification in dry areas, with adhesion based on suction cups or magnets. Hereafter, the main characteristics and features of a prototype proposed for holds inspection are focused and discussed, evaluating advantages and criticisms.

\section{Pneumatronic Climbing Prototype}

Internal inspections of marine structures and plants are a topic of specific interest for DREAMS Lab. It is currently oriented towards developing low cost, fully or semi-autonomous, simple and easily transportable modular kit units to be used as tools for the inspector leaving the task of selecting the most appropriate unit for a particular survey [23-25].

A distinguishing aspect of the followed design methodology of inspection units, in comparison to approaches followed by other researchers, is the research of simplicity and modularity. The unit is designed starting from a basic architecture, with the possibility of enhancement adding customized modules able to perform specific tasks. This approach is followed not only in the design of the unit described in this paper, but in all the designs of self-moving units under study for different inspection tasks in ship or off-shore units.

The fundamental necessary requirements to the unit can be synthesized as follows:

- easy and reliable working;

- capability of operation within hostile environments;

- simple programming;

- limited need for maintenance;

- on/off actuation, where possible;

- unsophisticated design;

- low cost;

- weight reduction.

Fig. 3 shows the basic geometry assumed as reference for the design: it consists of a cross-shaped structure with four symmetric arms connected to a central frame. Each arm is a double acting linear actuator, equipped at its mobile end with the attachment device to the surface, moved by single acting actuators.

In this way, the motion of the unit is the combination of motions along two orthogonal directions. This could be considered as an operative limit, though not significant if related to the motion required on surfaces such as the walls of holds.

Typical geometry of these walls is Greek-shaped (Fig. 4), with recurrent dimensions $\left(\Phi=63^{\circ}, \mathrm{d}_{\mathrm{cg}}=700\right.$ $\mathrm{mm}, \mathrm{b}_{\mathrm{f}-\mathrm{cg}}=700 \mathrm{~mm}, \mathrm{~b}_{\mathrm{w}-\mathrm{cg}:} 783 \mathrm{~mm}$; $\left.\mathrm{t}_{\mathrm{t}-\mathrm{net}:} 15 \mathrm{~mm}\right)$.

The self-moving unit is sized on these dimensions. Fig. 5 sketches the position of the unit on a vertical surface: fundamentals for the selection of the attachment devices are the quotes $a$ and $b$ as well as the position of the center of mass of the unit.

In order to define the basic architecture of the inspection unit, taking into account the variety of geometries present into a hold, the reference assumed is that of SKS tankers type (Fig. 6), where large areas can be inspected automatically but sporadic interventions of the operator are required for positioning the unit at the beginning of different paths.

These types of tankers have floor and walls that are flat in consequence to the double hull, imposed by IMO.

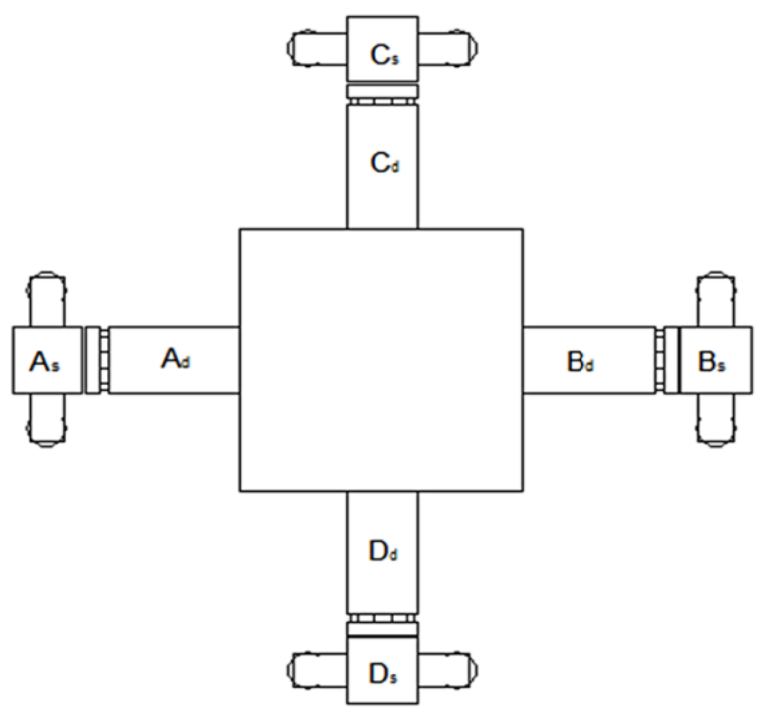

Fig. 3 Basic geometry of the climbing $\operatorname{robot}\left(A_{d}, B_{d}, C_{d}, D_{d}\right.$ : main double acting actuators. $A_{s}, B_{s}, C_{s}, D_{s}$ : secondary single acting actuators). 

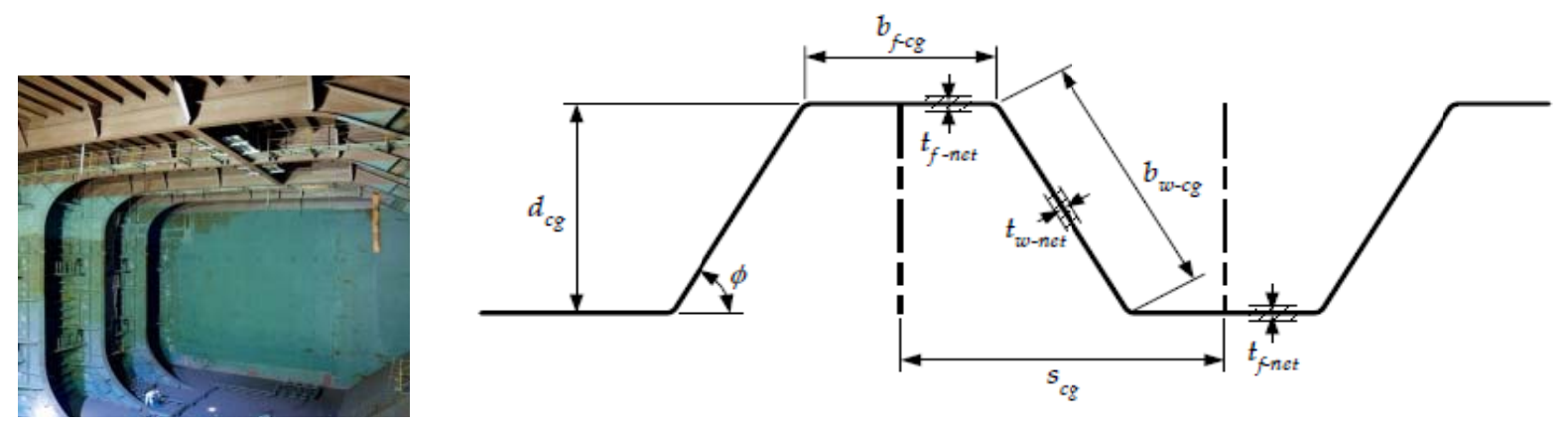

Fig. 4 Typical Greek-shape surface of hold walls.

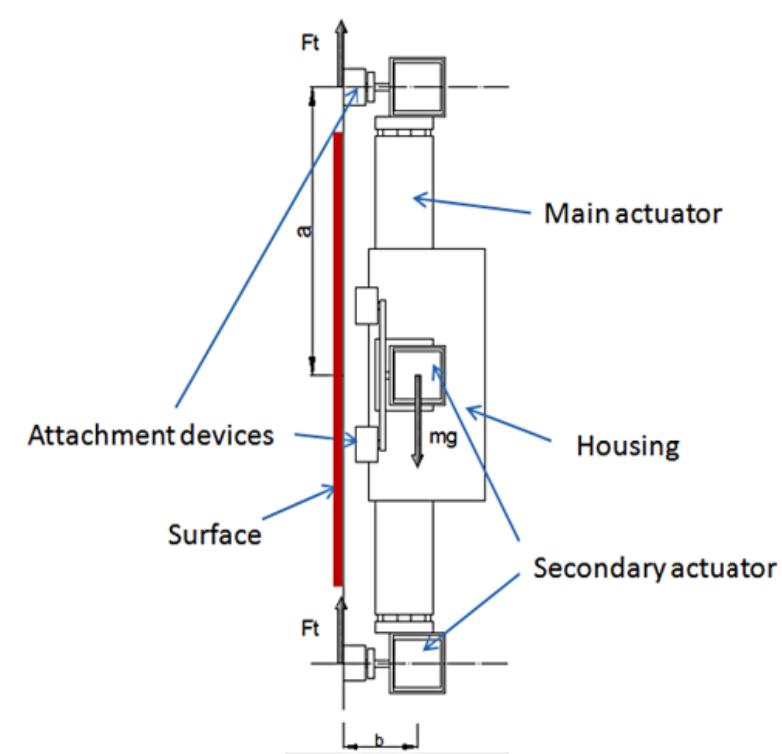

Fig. 5 Sketch of the unit on vertical surface.

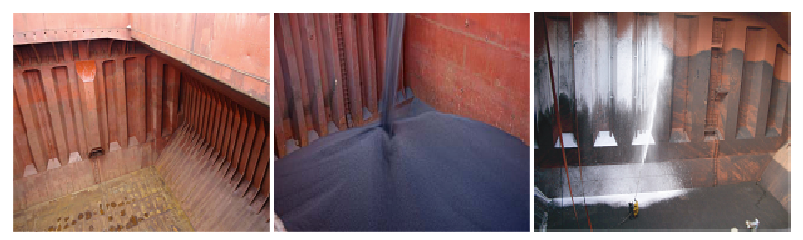

Fig. 6 Examples of SKS holds.

\section{Anchoring}

The problems related to the anchoring of the inspection unit to the surface under investigation, is subject of discussion among researchers, in particular with reference to technical products available on the market, prioritizing low cost solutions. Fundamental problems related to the surface, critical for the anchorage, are related to the presence of:

- polluting films (rust, oxides, environmental contaminating, absorbed gas films...);
- deposit of oil (greasy surface);

- mix between grease and dirt or grimes;

- welding beads;

- surface defects or local differences of surface finishing.

Possible methods of attachment involve:

- pneumatic suction cups;

- holding magnets;

- permanent magnetic strips;

- permanent magnetic walking beams;

- electrically settable permanent magnets;

- special magnet systems;

- spreading magnets.

Different methods of attachment require a careful comparison in order to select not necessarily the best solution but the most suitable one.

With reference to vacuum solutions, specific critical problems are related to metallic oily surfaces and to presence of welding beads. In fact, the friction coefficient between cup and oily surface is often strongly reduced with respect to the contact on clean surface.

Today friction cups specially designed for greasy surfaces allow the overcoming of this criticism. Thanks to the excellent grip on greasy surfaces, this type of suction cups can withstand high shearing strains, typical 2-4 times higher than with conventional suction cups. Some specific components are suitable for curved or flat surfaces and are manufactured in a specially developed material, with elastic properties like rubber and wearing qualities like polyurethane.

Problems related to positioning of suction cup over a 
welding bead can be solved using multiple suction cup devices instead of single suction cups, by designing the attachment device with sensors able to detect the presence of the welding, and to adjust automatically the device position in such a way the welding is positioned between two cups. In addition, further improvements can be reached using oval or rectangular suction cups, instead of the classical circular geometry.

With reference to magnetic attachment devices, criticisms concern the rigid structure of the magnet and the choice between permanent magnets and electro-magnets. Difficulties of adaptation of the magnet on irregular surfaces can be overcome using multiple magnets instead of one larger magnet. Permanent magnets simplify electrical wiring, but increase the value of the external applied force necessary to detach the magnet. Electro-magnetic solutions are more flexible, but require an automatic control of the activation/deactivation phases.

Both pneumatic and electrical attachments are considered in the design of the proposed unit: detailed designs have been developed for suction cups and electro-magnets.

\subsection{Pneumatic Anchoring}

Fig. 7 collects results of different types of suction cups in contact on steel surfaces: the cup is loaded with axial forces of 25, 50, 60 and $70 \mathrm{~N}$ and under vacuum pressures of 20, 40, 60 and $80 \mathrm{kPa}$. The anchoring condition is analyzed, distinguishing on five levels (from anchoring to immediate detaching). Surface, suction cups, and levels considered are:

Surfaces. S1: Steel; S2: Oiled Steel; S3: Oxidized Steel.

Suction Cups. SC1: Flat, Silicon, $\Phi 55 \mathrm{~mm}$; SC2: Bellows, Rubber, $\Phi 52$ mm; SC3: Bellows, Silicon, $\Phi$ 60 mm; SC4: Flat, NBR, $\Phi 110$ mm; SC5: Flat, NPV reinforced, $\Phi 110 \mathrm{~mm}$.

Levels. 5: Immediate detaching; 4: Detaching with delay; 3: Fully slipping; 2: Partial slipping; 0: Anchoring.
Of course, increasing the diameter of the suction cups (axial force being equal) the number of correct anchoring increases. Fig. 8 reports one of the results of test on silicon cups and round bellow cups, showing the deformation of the cup vs. the lateral force, under different vacuum pressure levels.

These tests have been related to detailed analyses oriented to evaluate the suction cup deformations and displacements. In particular, the lip in contact with the surface is simulated using finite elements approaches for different sizes, materials and geometries.

Fig. 9 shows examples of this phase of analysis, in particular with reference to SC1 suction cup, previously cited. The cup is loaded with a lateral force of $50 \mathrm{~N}$ corresponding to the condition of the climbing unit anchored on steel vertical surface with only one couple of suction cups. The vacuum pressure is -40 $\mathrm{kPa}$ : the lip is deformed but the suction cup maintains the anchorage.

The critical level of lateral force producing the detaching obviously depends to the size and to the geometry of the suction cup.

\subsection{Magnetic Anchoring}

Criticisms related to the application of permanent or electrical magnets concern the real forces generated within the attachment area and the real contact area, taking into account the rigid structure of the magnet. Solutions based on two or three magnets for each arm are studied and tested.

Table 1 reports the characteristics of six electro-magnets (two for three different producers, conventionally named A, B and C) and in Table 2 is collected a comparison among these magnets, coupled in groups.

Tests on slipping and overturning conditions are implemented on steel and oiled steel surfaces, distinguishing the static contact (fully anchoring) to the dynamic contact (beginning of slipping).

Fig. 10 reports the corresponding strain analyses for some of these anchorage solutions, showing the good 


\begin{tabular}{ll}
\hline Suction & Surface \\
Cup & S1
\end{tabular}

SC1

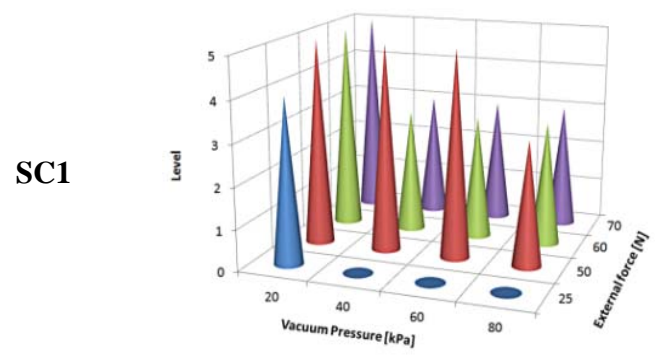

SC2
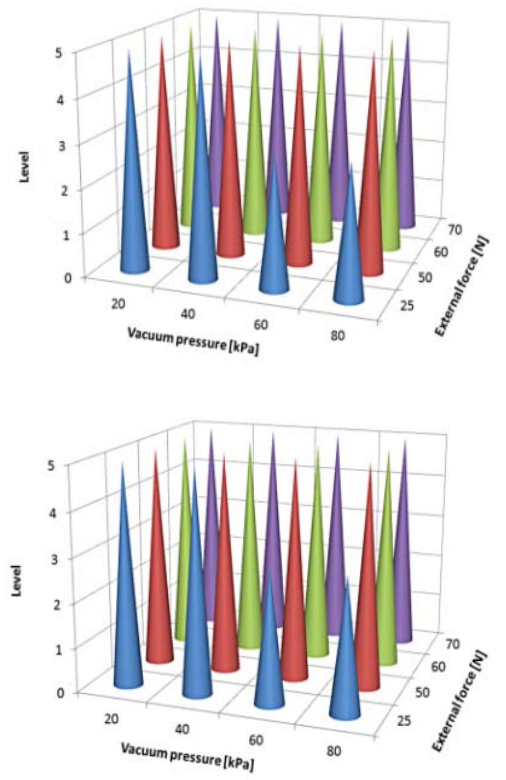

SC4
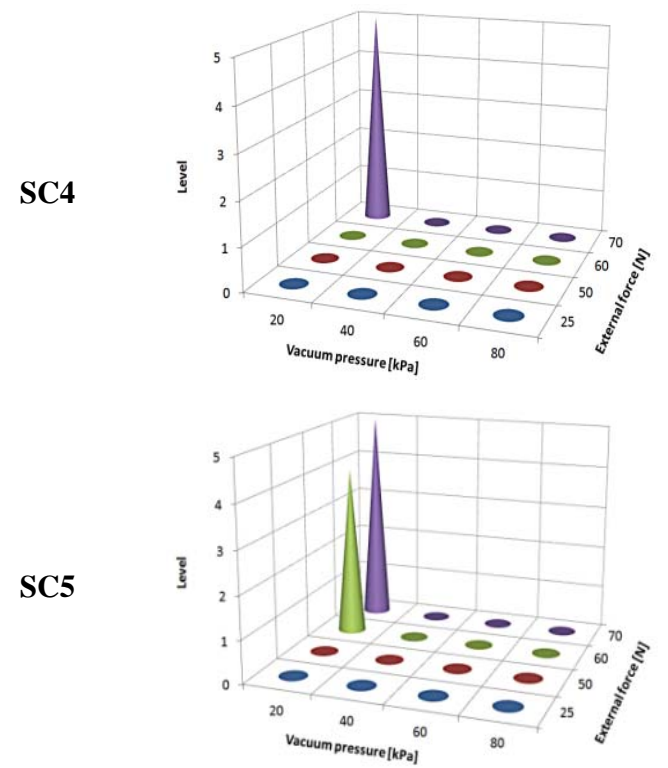

S2
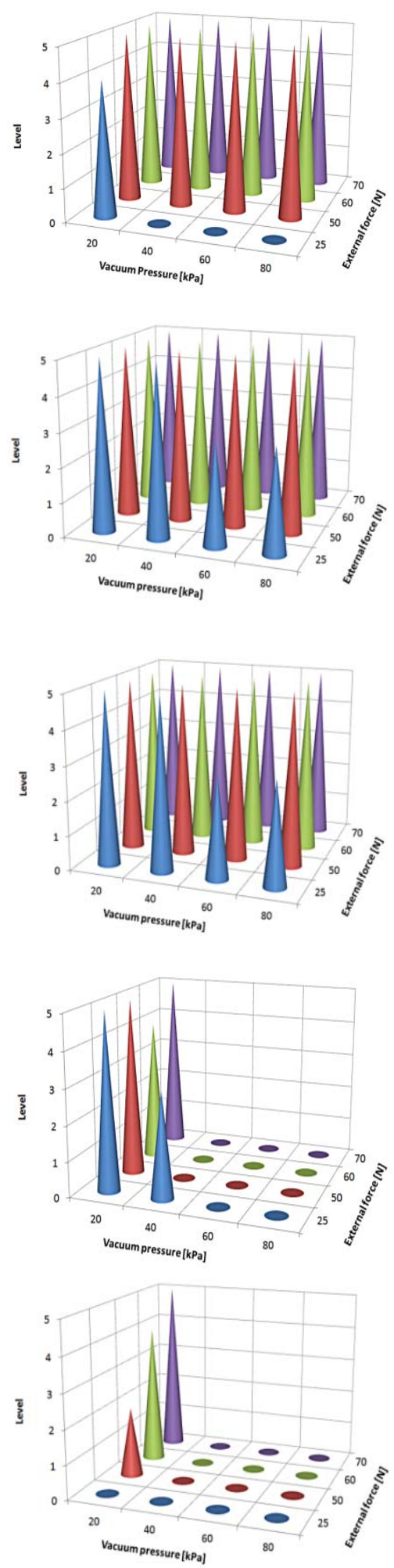
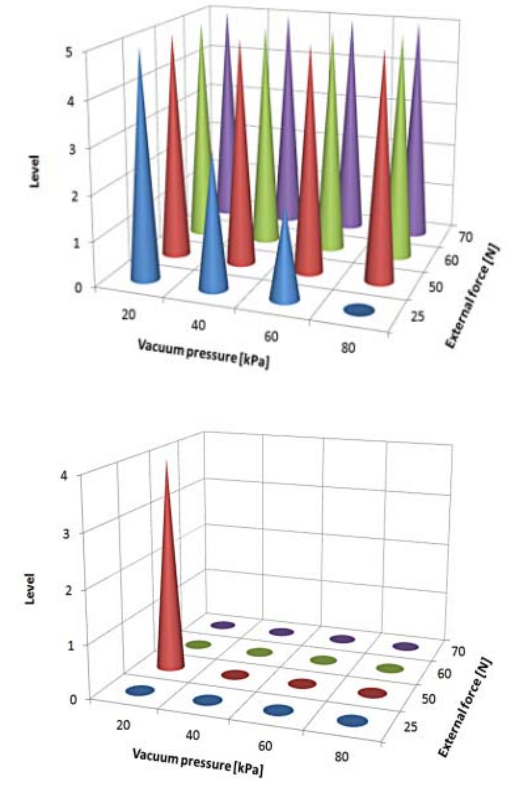

S3
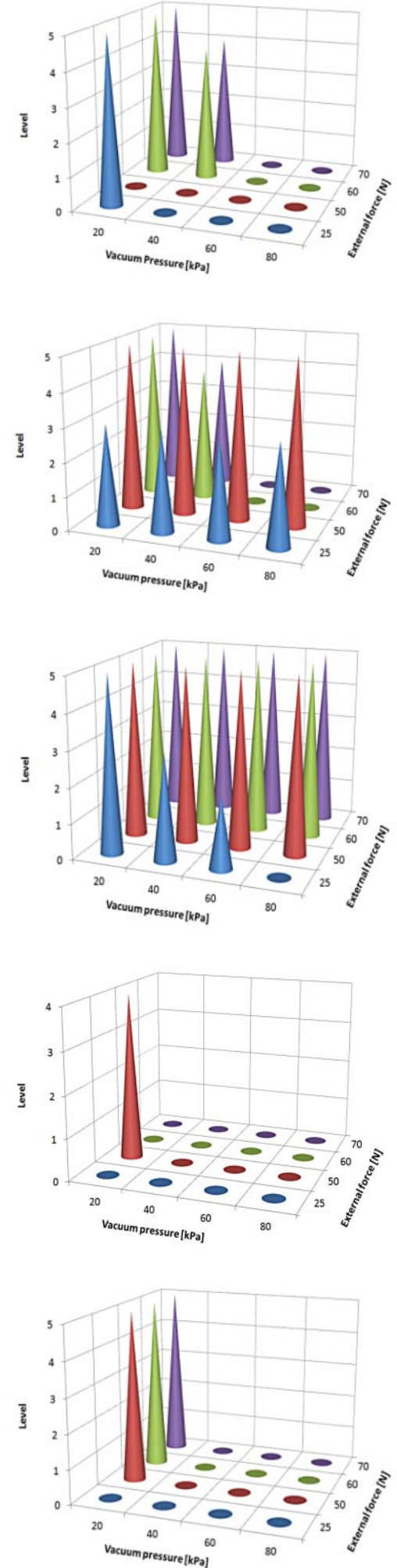

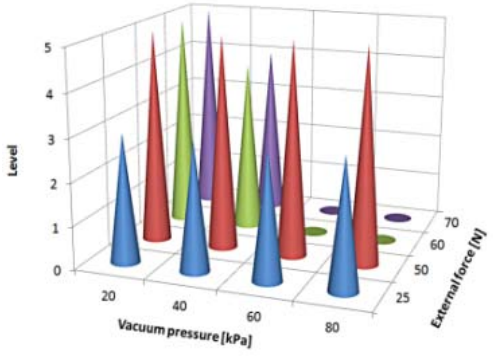

Fig. 7 Tests on suction cups in contact with steel surfaces. 

Low Cost Unit for Inspection of Holds

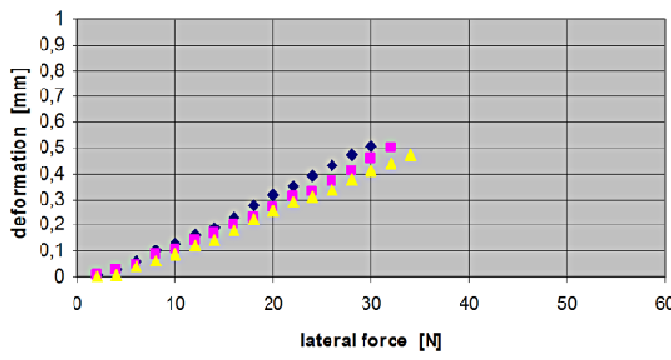

(a)

Fig. 8 Tests on silicon (a) and rubber (b) suction cups.

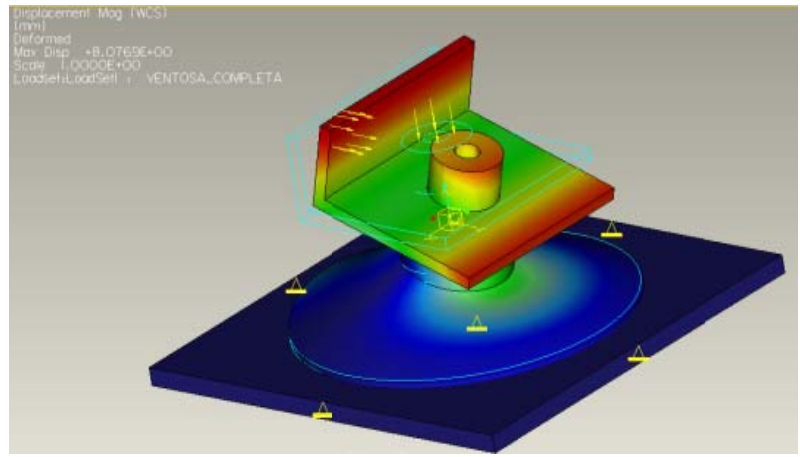

(a)

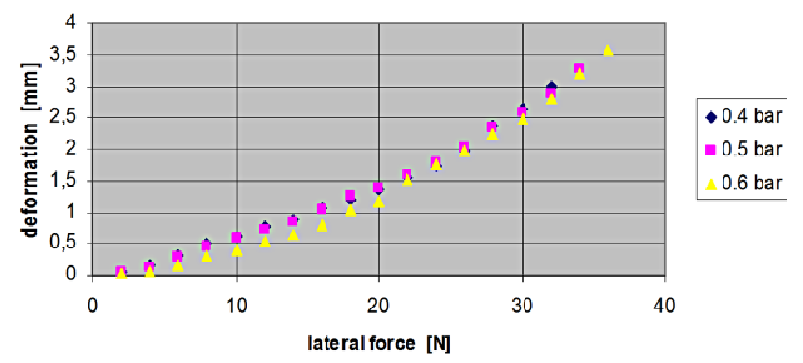

(b)

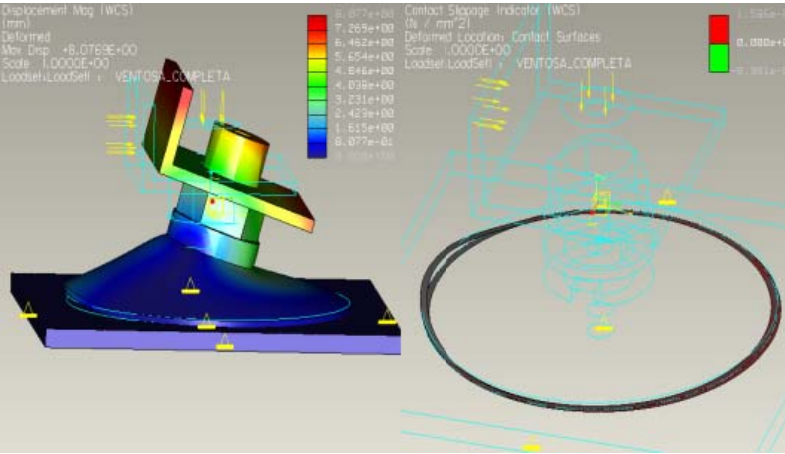

(b)

Fig. 9 3D model of a suction cup: deformation (a) and lip displacement (b).

Table 1 Electro-magnets characteristics.

\begin{tabular}{|c|c|c|c|c|c|}
\hline $\begin{array}{c}\text { Electro- } \\
\text { magnet }\end{array}$ & Voltage [Vcc] & Power [W] & $\begin{array}{c}\text { Diameter } \\
{[\mathrm{mm}]}\end{array}$ & $\begin{array}{c}\text { Anchoring } \\
\text { force }[\mathrm{N}]\end{array}$ \\
\hline A1 & 24 & 5 & 30 & 100 & 270 \\
\hline A2 & 24 & 7.5 & 39 & 300 & 440 \\
\hline B1 & 24 & 4.5 & 30 & 85 & 200 \\
\hline B2 & 24 & 5 & 40 & 250 & 600 \\
\hline C1 & 24 & 4.1 & 35 & 160 & 150 \\
\hline C2 & 24 & 4.4 & 45 & 290 & 290 \\
\hline
\end{tabular}

behavior of this rigid anchoring. The clamp is subject to a maximum stress of $47 \mathrm{MPa}$ (Fig. $10 \mathrm{~b}$ ), that decreases to $18 \mathrm{MPa}$ adopting the configuration with three magnets.

\section{Model of the Structure}

The concept and the design of the unit take into account four different possible configurations:

- pneumatic attachment with external fluid power and electrical supplies;

- pneumatic attachment with fluid power and electrical supplies on board;

- magnetic attachment with external electrical power supply;

- magnetic attachment with on board electrical power supply.

Solutions with external power supplies are simplified and lighter but show the disadvantage of the 
Kit of Self-Moving Units for Automatic Inspection of Marine Structures and Plants: Low Cost Unit for Inspection of Holds

Table 2 Anchoring analyses on electro-magnets.

\begin{tabular}{|c|c|c|c|c|c|c|}
\hline \multirow{3}{*}{$\begin{array}{l}\text { Electro- } \\
\text { magnet }\end{array}$} & \multirow{3}{*}{$\begin{array}{l}\text { No. of magnets } \\
\text { per anchorage }\end{array}$} & \multicolumn{4}{|c|}{ Slipping test } & \multirow[t]{3}{*}{ Overturning test } \\
\hline & & \multicolumn{2}{|c|}{ Steel } & \multicolumn{2}{|c|}{ Oiled steel } & \\
\hline & & Static contact & $\begin{array}{l}\text { Dynamic } \\
\text { contact }\end{array}$ & $\begin{array}{l}\text { Static } \\
\text { contact }\end{array}$ & $\begin{array}{l}\text { Dynamic } \\
\text { contact }\end{array}$ & \\
\hline $\mathrm{A} 1$ & 2 & $\sqrt{ }$ & $\sqrt{ }$ & $\otimes$ & $\otimes$ & $\sqrt{ }$ \\
\hline $\mathrm{A} 1$ & 3 & $\sqrt{ }$ & $\sqrt{ }$ & $\sqrt{ }$ & $\otimes$ & $\sqrt{ }$ \\
\hline A2 & 2 & $\sqrt{ }$ & $\sqrt{ }$ & $\otimes$ & $\otimes$ & $\sqrt{ }$ \\
\hline B1 & 3 & $\sqrt{ }$ & $\sqrt{ }$ & $\otimes$ & $\otimes$ & $\sqrt{ }$ \\
\hline B2 & 2 & $\sqrt{ }$ & $\sqrt{ }$ & $\sqrt{ }$ & $\otimes$ & $\sqrt{ }$ \\
\hline $\mathrm{C} 1$ & 3 & $\sqrt{ }$ & $\sqrt{ }$ & $\otimes$ & $\otimes$ & $\sqrt{ }$ \\
\hline $\mathrm{C} 2$ & 2 & $\sqrt{ }$ & $\sqrt{ }$ & $\otimes$ & $\otimes$ & $\sqrt{ }$ \\
\hline \multicolumn{7}{|c|}{$\sqrt{ }$ Verified; $\otimes$ Not verified } \\
\hline
\end{tabular}

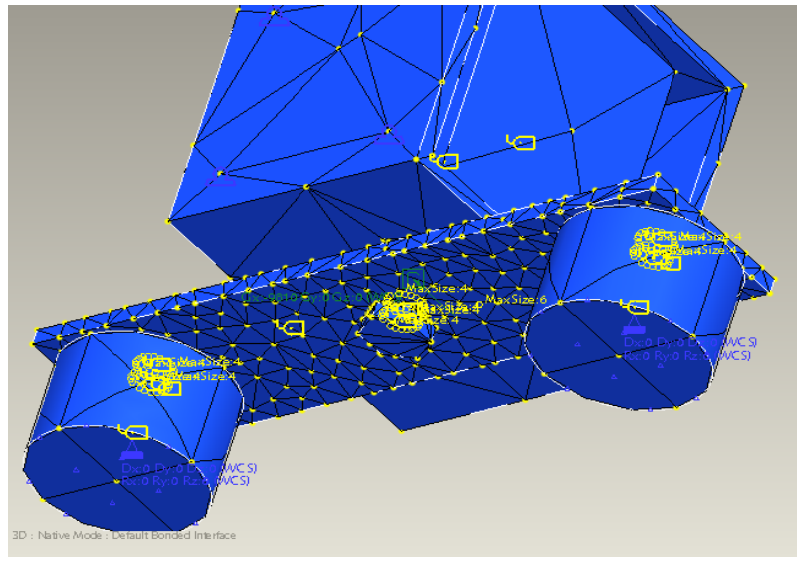

(a)

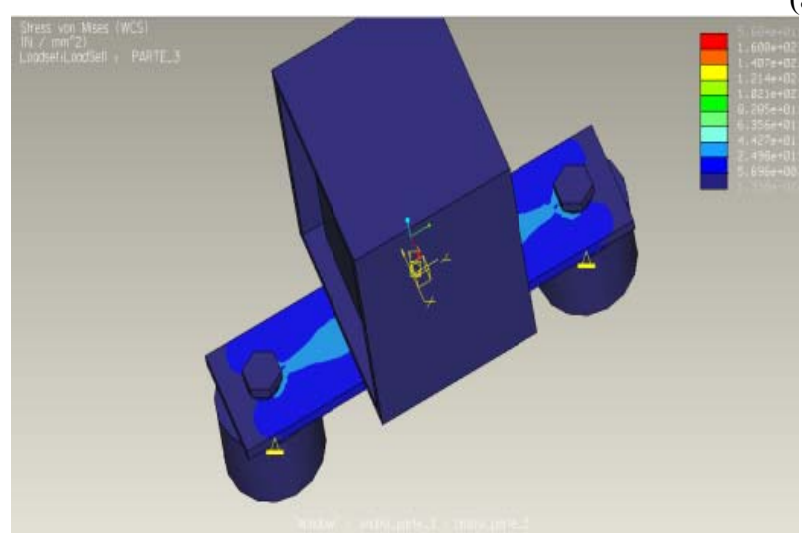

(b)

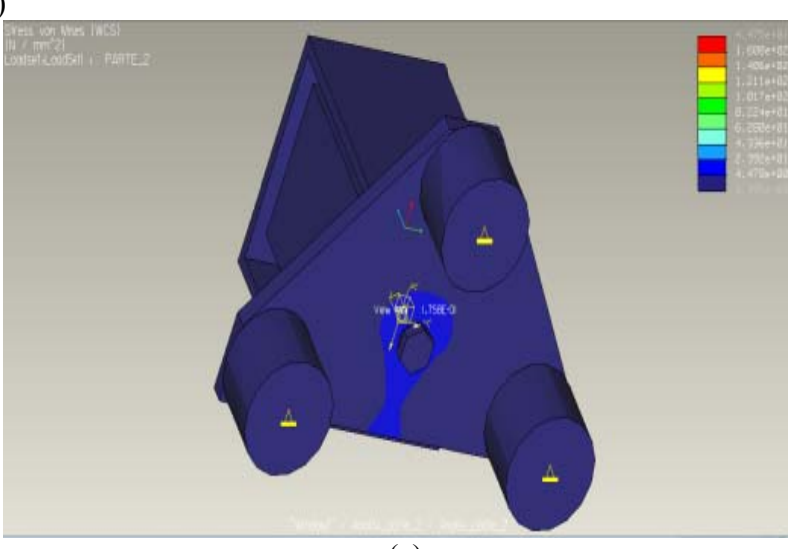

(c)

Fig. 10 3D models of magnets: geometries with two (a and b) or three (c) magnets. 
umbilical cord. It limits the path and the dexterity of the unit but can be acceptable in specific confined spaces.

Solutions with embedded power supplies require the realization of an air-compressed reservoir on board and the installation of batteries having a sufficient autonomy to allow a practical use of the unit. Fig. 11 shows the case of embedded reservoir realized in the central body of the climbing unit. The analysis of stresses and strains is developed using a 3D model of the structure implemented in Pro-Engineer environment, under different constraint conditions, as shown in Fig. 12. Main settings concern Young's modulus Poisson's coefficient for the materials of the different parts of the unit (Body: steel, $\mathrm{E}=199,948$ $\mathrm{MPa}, v=0.27$. Actuators and clamps: aluminum, $\mathrm{E}=$ 73,084 MPa, $v=0.33$ ). The constraint characteristics are validated with experimental tests in presence of different kinds of surfaces and different superficial

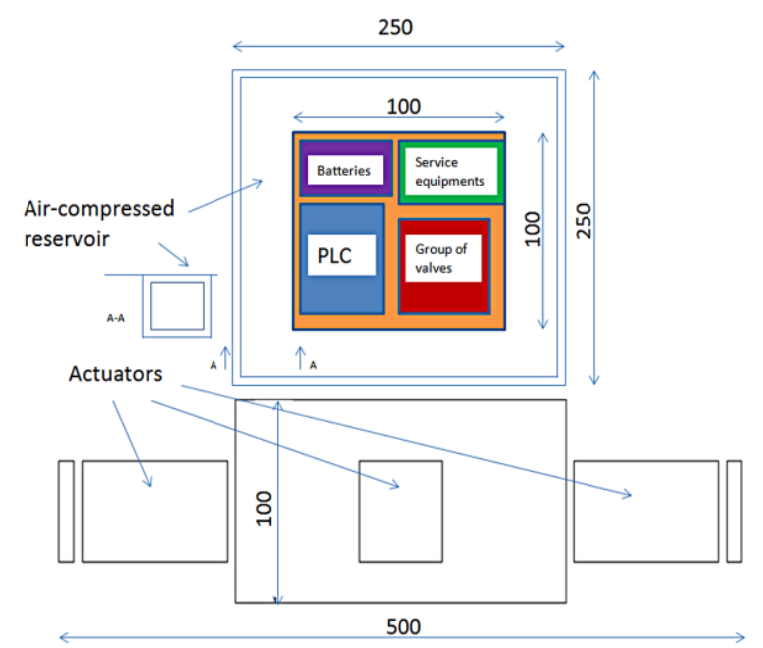

Fig. 11 Sketch of structure (top and side views) with embedded devices (quotes in $\mathrm{mm}$ ).

Case 1: Top constraint

Case 2: Bottom constraint

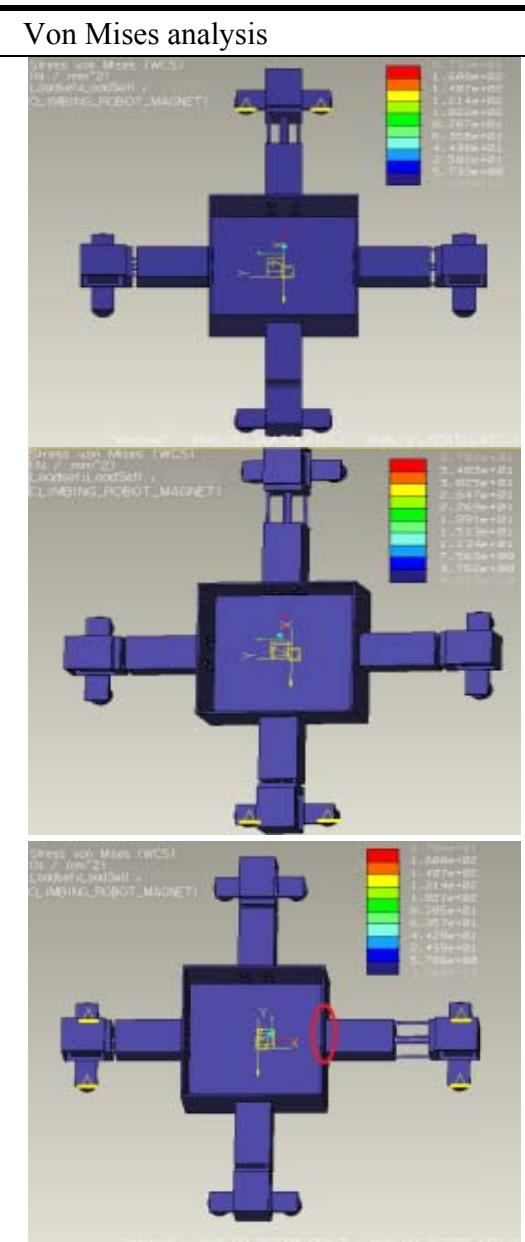
Displacements

Case 3: Lateral constraint

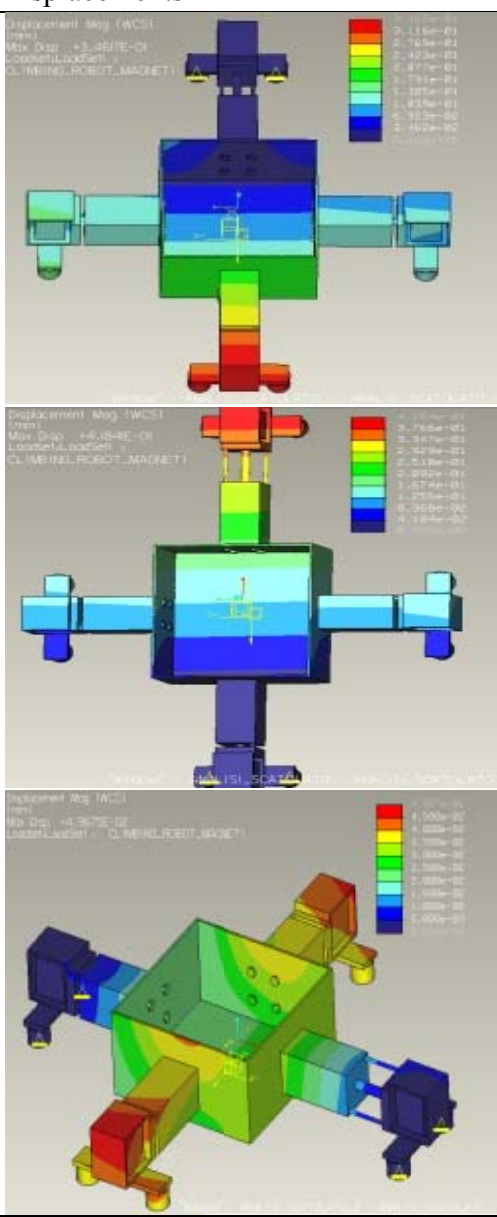

Fig. 12 Stress and strain analyses. 
status (clean, wet, oily, and dirty). In the case 1 (anchorage of the unit in correspondence of the $\mathrm{C}_{\mathrm{s}}$ actuator, in Fig. 1) the maximum value of strength is 30 $\mathrm{MPa}$, significantly less than the admissible limit of the material (aluminum, $160 \mathrm{MPa}$ ); the maximum displacement detected is $0.34 \mathrm{~mm}$. In the case 2 the unit is anchored in correspondence to the actuator $\mathrm{D}_{\mathrm{s}}$ (Fig. 1) and the structure in stressed to compression: the maximum value of strength is $34 \mathrm{MPa}$ and the maximum displacement is $0.4 \mathrm{~mm}$. Finally (case No. 3), the structure of the climbing unit is studied with connections at the ends of the arms A and B (Fig. 1): this is the most favorable condition, with minimum displacements $(0.05 \mathrm{~mm})$. On the whole, the implemented model fully satisfies the strength and displacement limits. It is used as reference to the design of the prototype hereafter described.

\section{Electronic Control System and Equipment}

A micro-PLC installed on board manages the motion control. The basic architecture of the unit plans that all the on-off signals drive the actuations: having consider that the pneumatic solution is a group of micro-valve (Fig. 11) that drive the main actuators and the ejectors. PLC input signals are acquired by position sensors and by vacuum gauges.

The unit can be programmed to realize different paths, as shown in Fig. 13, taking into account the shape of the hold surfaces. More sophisticated geometries can use flow-rate or pressure proportional valves in order to generate steps defined by the user. In this case, remote wireless command by joystick can be applied.

Typical equipment mounted on the unit to carry out the non-destructive inspection tasks are:
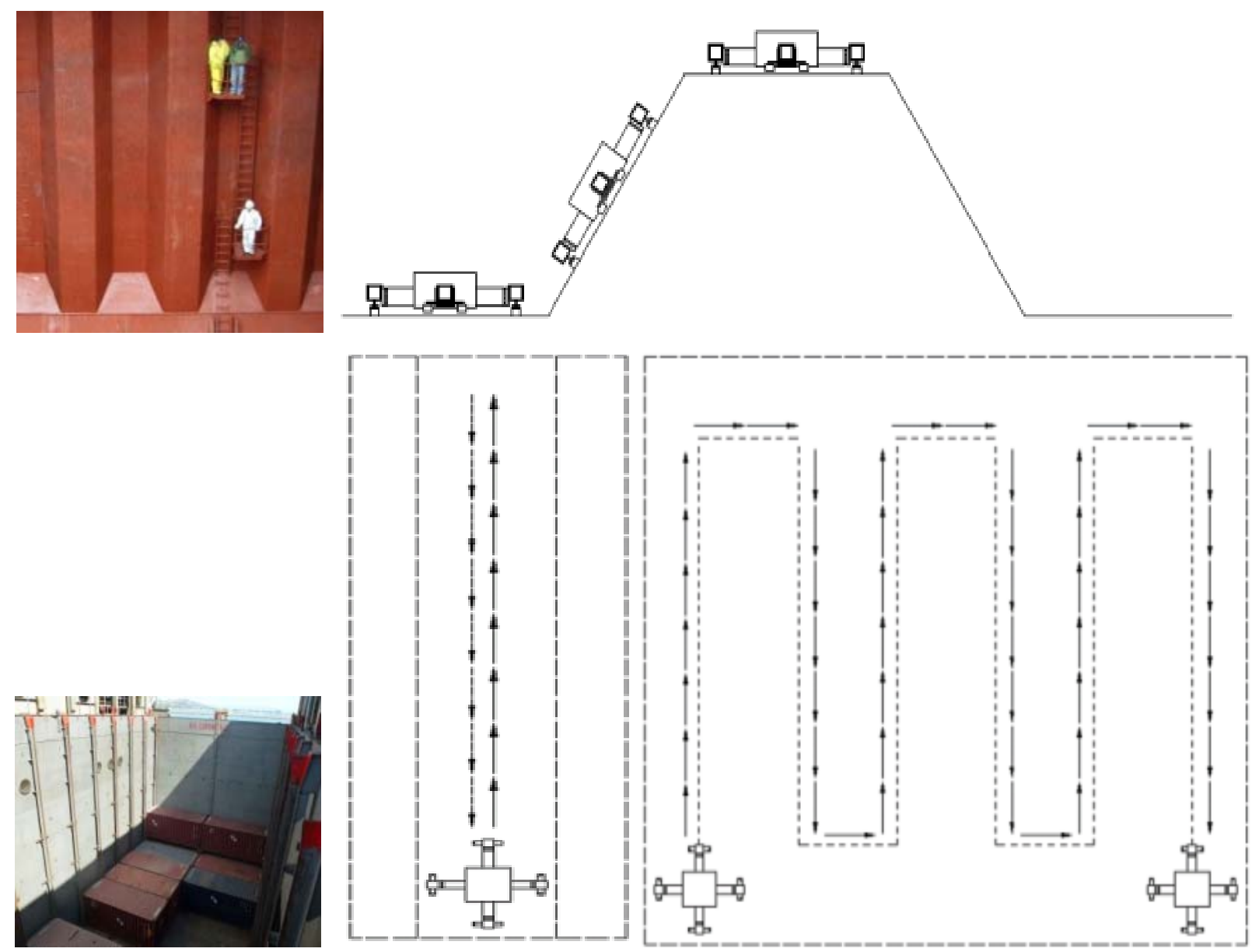

Fig. 13 Typical programmable paths. 

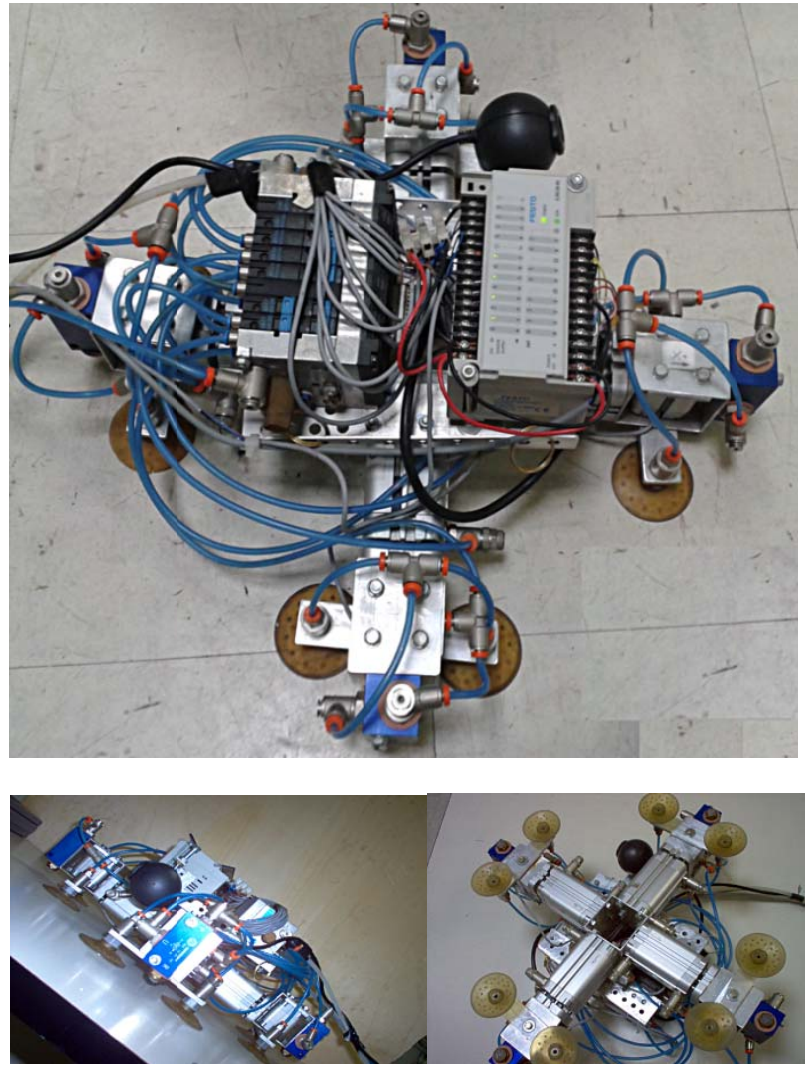

Fig. 14 Prototype of pneumatic climbing unit.

- micro video-cameras (wireless or with integrated battery), to obtain a complete mapping of the inspected surface;

- micro thermo-cameras, to detect defects also in presence of puttying or painting;

- thickness ultrasonic measurement devices, detecting the state of the steel plates.

\section{Experiments}

In order to test the performances and the limitations of the proposed unit a basic prototype has been constructed and tested. The self-moving unit is able to climb on flat or low curvature surfaces, generically oriented. It is fully pneumatic, with four orthogonal crossed main actuators: each of them carries, at its end, another cylinder able to lift and to lower a couple of suction cups (Fig.14). Pneumatic valves drive also the ejectors supporting the suction cups; in order to reduce the weigh and to simplify the pneumatic connections, the driving system is realized as a group of compact pneumatic valves controlled by a micro-PLC. All the components are embedded on the robot; in this way, the "umbilical cord" connecting the autonomous system to the frame is reduced to a single hose and to a single electrical wire ( $24 \mathrm{~V}$ power supply), increasing the robot dexterity.

Motion tests on different kinds of surfaces have been developed; climbing operations on horizontal, tilted and vertical surfaces have been programmed, while also changing the surface roughness and the motion sequence of the robot. About the roughness of steel plates the reference assumed is the European Standard UNI EN 10130, classifying the products in four classes:

$$
\begin{aligned}
& \text { bright, } \mathrm{Ra} \leq 0.4 \mu \mathrm{m} \\
& \text { semi bright, } \mathrm{Ra} \leq 0.9 \mu \mathrm{m} \\
& \text { standard, } 0.6 \mu \mathrm{m}<\mathrm{Ra} \leq 1.9 \mu \mathrm{m} \\
& \text { rough, } \mathrm{Ra}>1.6 \mu \mathrm{m}
\end{aligned}
$$

Experimental tests have been mainly developed on standard plates. Climbing motions on steel, oiled steel, oxidized steel, glass, plastics, rolled wood, plasters have been successfully tested.

The structure of this type of climbing robot is rigid: nevertheless, specific tests have shown the possibility to use it not only on flat surfaces, but also on low curvature walls (maximum radius of curvature 1,700 $\mathrm{mm}$ ), thanks to the local deformations of the suction cups. The overall dimensions of the proposed prototype are $600 \times 600 \times 150 \mathrm{~mm}$, and the weight is $5.5 \mathrm{~kg}$. Step sequences are completely programmable. The gripping is assured by the control of the vacuum level inside the cups: if the level decreases below- 0.8 bar the unit stops itself, maintains the position and generates an air-raid warning. The average speed when the unit is climbing is about $0.1 \mathrm{~m} / \mathrm{s}$ : the examination by video-camera is continuous, while examinations by others devices (e.g. UTM) require additional times related to the specific measurement.

The prototype has shown good technical reliability: cycles up to one thousand steps with U-turns have been realized without problems. The working performance 

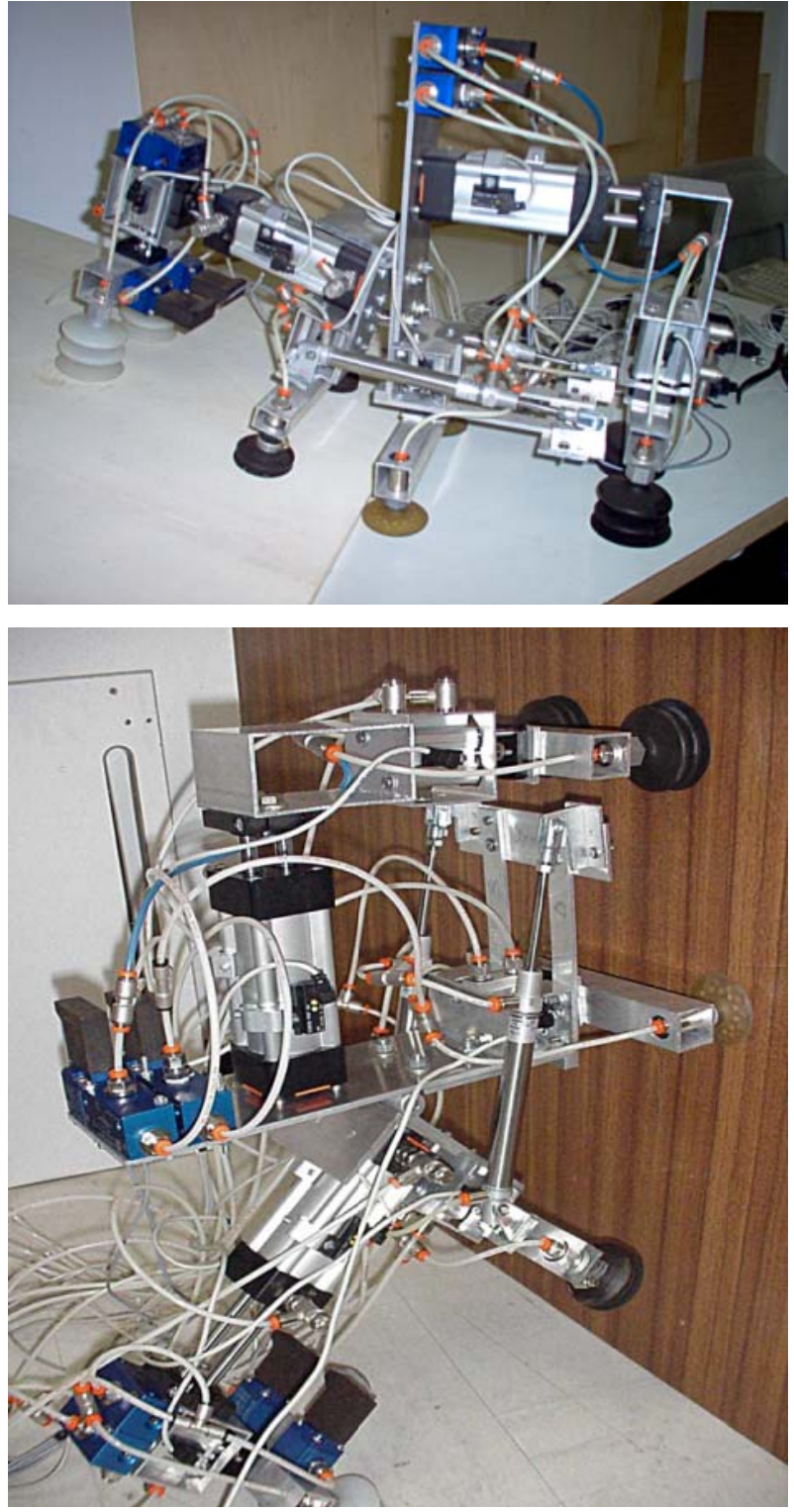

Fig. 15 Articulated self-moving unit.

has been tested monitoring the real deformation of the structure by means of strain gauges: taking into account the suction cup deformations, the results are comparable with those obtained from the numerical model. These results have been useful to validate the numerical model, generating a parametric tool applicable in the study of new units.

Structural improvements could be studied passing from the prototype to industrialized unit, in particular protecting electronic and pneumatic devices from aggressive environments and from unexpected impacts.

\section{Alternative Geometries}

The corresponding prototype shows good performances on flat or low curvature surfaces, but it is not able to move itself on surfaces having variable curvatures. Fig. 15 shows an alternative geometry designed and tested: it consists on an articulated unit using the same types of anchoring devices but having an articulated geometry. It is able to move itself on a surface and automatically recognize the slope variation and modifying the position of the front following the surface slope.

The unit shown in Fig. 15 allows climbing motions only in a single direction: it consists of two parts (front and back) respectively mounted on articulated frames. If, during the motion of the robot, the slope of the surface varies, a contact less sensor detects this variation and the front part rotates automatically. This alternative solution includes an interesting improvement of motion control, substituting the point-to-point control (by PLC) with a distributed control (Actuator Sensor Interface, AS-I). This approach allows the elimination of the PLC on board but limits the use to digital input/output signals. Micro-piezoelectric proportional valves are still under test in another alternative solution designed to enhance the flexibility of control by remote users.

\section{Concluding Remarks}

Ship inspection is a very real problem, involving aspects of cost, personnel employment, safety, and instrumentation. It is currently performed manually and there are not general rules or criteria able to decide whether automatic approaches can substitute, entirely or partially, the traditional methods. Anyway self-moving units are attractive to automate several inspection phases.

A low cost climbing self-moving unit oriented to inspection tasks is proposed. A parametric model able including sub-models of the structure and of the anchoring devices (suction cups or electro-magnets) is developed. The corresponding prototype shows good 

Low Cost Unit for Inspection of Holds

performances on flat or low curvature surfaces, but it is not able to move itself on surfaces having variable curvatures. Articulated variations can be evaluated, taking into account the increasing mechanical complexity.

The prototype under study is equipped with basic instrumentation, but further measurement devices can be easily placed on board. In particular, at the moment the prototype is not equipped with surface-recognition tools: but considering that it may be easier that the robot's eye detects surface failures as it may be harder for the human operator to be able to wholly appreciate defects on surfaces through the use of conventional cameras, this aspect will be surely considered in further development of the unit.

Other experiences, still under development, are oriented to eliminate the umbilical cord, installing on board a micro-compressor and batteries for electric power supply. The unit takes part of a low cost and user-friendly kit of survey, conceived to support inspectors during periodic visits on board of ships and offshore platforms; next papers will describe solutions proposed to inspection of ducts or pipes and confined spaces.

\section{References}

[1] Kim, S., Asbeck, A. T., Cutkosky, M. R., and Provancher, W. R. 2005. "Spinybot II: Climbing Hard Walls with Compliant Microspines." Proc. of the 2005 IEEE International Conference on Robotics and Automation: 601-6.

[2] Balaguer, C., Virk, G., and Armada, M. 2006. "Robot Applications against Gravity." IEEE Robotics and Automation Magazine 13 (1): 5-6.

[3] Longo, D., and Muscato, G. 2004. "A Modular Approach for the Design of the Alicia3 Climbing Robot for Industrial Inspection.” Industrial Robot: An International Journal 31 (2): 148-58.

[4] Murphy, M. P., Tso, W., Tanzini, M., and Sitti, M. 2006. "Waalbot: An Agile Small-Scale Wall Climbing Robot Utilizing Pressure Sensitive Adhesives." Proc. of the 2006 IEEE/RSJ International Conference on Intelligent Robots and Systems 12 (3): 3411-6.

[5] Kim, H., Kim, D., Yang, H., Lee, K., Seo, K., Chang, D., and Kim, J. 2006 "A Wall Climbing Robot with Vacuum Caterpillar Wheel System Operated by Mechanical Valve.”
Proc. of the 9th International Conference on Climbing and Walking Robots, 28-33.

[6] Backes, P. G., Bar Cohen, Y., and Joffe, B. 1997 "The Multifunction Automated Crawling System (MACS)." Proc. of the 1997 IEEE International Conference on Robotics and Automation, 335-40.

[7] Rosa, G. L., Messina, M., Muscato, G., and Sinatra, R. 2002. "A Low-Cost Lightweight Climbing Robot for the Inspection of Vertical Surfaces." Mechatronics 12 (1): 71-96.

[8] Tso, S. K., Fan, K. L., Fung, Y. H., Han, L., Tang, D. W., Liu, K. P., and Tong, F. 2003. "Inspection of Large Tile Walls for Highrise Buildings Based on a Mechatronic Mobile System." Proc. of the 2003 IEEE/ASME Intl. Conference on Advanced Intelligent Mechatronics, 669-72.

[9] Xiao, J., Xiao, J., Xi, N., and Sheng, W. 2004. "Fuzzy Controller for Wall-Climbing Microrobots." Proc. of the 2004 IEEE International Conference on Robotics and Automation, 5033-8.

[10] Elkmann, N., Kunst, D., Krueger, T., Lucke, M., Bohme, T., Felsch, T., and Sturze, T. 2004. "SIRIUSc: Facade Cleaning Robot for a High-Rise Building in Munich, Germany." Proc. of the 7th International Conference Climbing and Walking Robots, 1033-40.

[11] Hirose, S., Nagakubo, A., and Toyama, R. 1991. "Machine That Can Walk and Climb on Floors, Walls and Ceilings." Proc. of the International Conference on Advances in Robotics, 753-8.

[12] Pack, R. T., Christopher, J. L., and Kawamura, K. 1997. "A Rubbertuator-Based Structure-Climbing Inspection Robot." Proc. of the 1997 IEEE International Conference on Robotics and Automation, 1869-74.

[13] Faina, A., Souto, D., Deibe, A., Lopez-Pena, F., Duro, R. J., and Fernandez, X. 2009. "Development of a Climbing Robot for Grit Blasting Operations in Shipyards." Proc. IEEE Intl. Conf. on Robotics and Automation, 200-5.

[14] Han, S. C., Kim, J., and Yi, H. C. 2009. "A Novel Design of Permanent Magnet Wheel with Induction Pin for Mobile Robot.” Int. J. Precis. Eng. Manuf. 10 (4): 143-6.

[15] Aracil, R., Saltaren, R. J., and Reinoso, O. 2006. "A climbing Parallel Robot.” IEEE Robotics and Automation Magazine 13 (1): 16-22.

[16] Vona, M., Detweiler, C., and Rus, D. 2006. "Shady: Robust Truss Climbing with Mechanical Compliances." Proc. of the 2006 International Symposium of Experimental Robotics, 431-40.

[17] Warszawski, A. 1999. Industrialized and Automated Building Systems-A Managerial Approach, 2nd ed. Taylor \& Francis.

[18] Zhang, H., Zhang, J., Liu, R., and Zong, G. 2005. "Realization of a Service Robot for Cleaning Spherical 
Surfaces.” Advanced Robotic Systems 2 (1): 53-8.

[19] Menon, C., and Sitti, M. 2006. "A Biomimetic Climbing Robot Based on the Gecko.” Bionic Engineering 3 (3): 115-25.

[20] Cho, K. J., Koh, J. S., Kim, S., Chu, W. S., Hong, Y., and Ahn, S. H. 2009. "Review of Manufacturing Processes for Soft Biomimetic Robots.” Int. J. Precis. Eng. Manuf. 10 (3): 171-81.

[21] Bibuli M., Bruzzone G., Caccia M., Giacopelli, M., Petitti A., and Spirandelli, E. 2012. "MARC: Magnetic Autonomous Robotic Crawler Development and Exploitation in the MINOAS Project." 11th International Conference on Computer and IT Applications in the Maritime Industries.
[22] Bibuli, M., Bruzzone, G., and Caccia, M. 2011. "Robot Task Allocation and Path-planning Systems in the MINOAS Project Framework." 19th Mediterranean Conference on Control and Automation.

[23] Ravina, E. 2007. "A Pneumatic Climbing Robot for Inspection Tasks." 10th Scandinavian International Conference on Fluid Power (SICFP07), 25-34.

[24] Ravina, E. 2010. "A Low Cost Pneumatronic Unit for Pipes Inspections." Proceedings of the ASME 2010 10th Biennial Conference on Engineering Systems Design and Analysis.

[25] Ravina, E. 2015. "Self-moving Units for Inspection Tasks of Marine Structures and Plants." Proc. of NAV2015, 18th Intl. Conf. On Ships and Shipping Research, 601-10. 